\title{
Multi-drug Resistance and Extended-Spectrum $\beta$-Lactamase Production in Uropathogens from Hospitalized Patients in Gurugram, India
}

\author{
Rishabh Rajput ${ }^{1,2 *}$ and Surendra Sarsaiya ${ }^{1,3}$ \\ ${ }^{1}$ Department of Microbiology, Sri Satya Sai University of Technology and Medical Sciences, \\ Sehore-Madhya Pradesh, India \\ ${ }^{2}$ Department of Microbiology, Modern Diagnostic \& Research Centre, Gurugram, \\ Haryana, India \\ ${ }^{3}$ Key Laboratory of Basic Pharmacology of Ministry of Education, Zunyi Medical University, \\ Zunyi, Guizhou, China \\ *Corresponding author
}

\section{Keywords}

UTI, Multi-drug resistance,

Extended-Spectrum $\beta$-lactamase, ESBL,

E. coli, $K$.

pneumoniae, CLSI, ATCC,

Article Info

Accepted:

15 January 2018

Available Online:

10 February 2018
A B S T R A C T

Urinary tract infections (UTI) are one of the most frequent problems in hospitalized patients. Infections caused by the gram-negative bacteria which produces Extended-spectrum $\beta$-lactamases (ESBL) have become the severe problem in the hospitals across the globe. Increase in extended-spectrum betalactamase producing organisms in recent years has led to the limitation of treatment option. This study was designed to evaluate the occurrence of different uropathogens producing ESBL, its antibiogram and Multi-drug resistance (MDR) status in hospitalized patients. 885 non-repetitive urine samples were collected in the study. A total of $293(33.11 \%)$ samples showed significant bacteriuria. Females were found to be more infected as compared to males. The senior citizens were found to be most affected ( $>60$ years). The most common ESBL producing uropathogens were Escherichia coli (E. coli) followed by Klebsiella pneumoniae (K. pneumoniae). All the ESBL positive isolates were found to be MDR. The prevalence of MDR was also significantly increased due to ESBL production. The monitoring of antibiotic resistance and susceptibility of bacterial strains should be mandatory due to the higher frequency of the ESBL producing uropathogens found in the hospitalized patients. It was proved in this study that the multi-drug resistance was responsible for the significant treatment failure. Hence testing of ESBL production in the uropathogens is warranted. It is also suggested not to prescribe any antibiotics without undertaking the culture and sensitivity report as it may further erode the antibiotics sensitivity in the studied regional population. 


\section{Introduction}

UTIs are one of the most frequent problems in hospitalized patients ${ }^{1}$. Infections caused by the gram-negative bacteria which produces ESBL have become the severe problem in the hospitals across the globe. About 400 different types of ESBLs have been found around the world, among which TEM and SHV were more prevalent ${ }^{2}$. Mutations occurring in the genes encoding these enzymes are responsible for the formation of ESBLs ${ }^{3}$.

Globally, the outbreaks of infection in various hospitals have been supplanting by endemicity of ESBL producing strains. This may lead to increased patient mortality when antibiotics inactive against ESBL-producers are used ${ }^{4}$. ESBL producing organisms are those that hydrolyse the oxyimino beta-lactams and monobactams but have no effect on the cephamycins and carbapenems. Cephalosporins have been used for the treatment of Gram-negative bacterial infections since 1980 's ${ }^{5}$. Unfortunately, nowadays, beta-lactamase resistance has been growing among members of Enterobacteriaceae, including E. coli and $K$. pneumoniae. The most frequent cause of betalactam resistance is beta-lactamase enzymes, which disable beta-lactam drugs by breaking down the beta-lactam $\operatorname{ring}^{6}$. K. pneumoniae are the opportunistic pathogenic organism responsible for the different infections such as pneumoniae, UTIs and septicemia in both hospitalized and community environments ${ }^{7}$. Beta-lactams drugs which have a beta-lactam ring in their composition are used rampantly to save the lives of the patients with bacterial diseases $^{8}$.

Resistant bacteria are emerging across the world as a threat to favourable outcomes of treatment of common infections in the community as well as hospital-acquired infections. Urinary tract, pyogenic infections, respiratory infections and gastrointestinal infection are the most common infections caused by a family of Enterobacteriaceae. Among this family, E. coli has been the most commonly isolated pathogen. It is very well known to exhibit multidrug resistance. Prolonged or undesirable antibiotic therapy, overstay in the hospitals and nursing homes, severe illness, unlimited use of thirdgeneration cephalosporins, fluoroquinolones, and increased use catheters are crucial risk factors for infections with MDR resistant Escherichia coli ${ }^{9}$.

It is essential to understand that there may be the marked differences in the antibiogram between different geographic locations within the big country like India. Since most of the UTIs treated empirically, the selection of the antibiotics should be determined, not only by the predicted sensitivity profile. Thus, the knowledge of the local antibiogram of the common uropathogens is essential for the prudent empiric therapy. Therefore, with the reports on the high frequency of MDR and ESBL formation amongst the E. coli from different part of the India ${ }^{9,10}$ and the paucity of information on their antibiogram, especially of the uropathogens from Gurugram-Haryana, an northern region in India, the present study was done to characterize the uropathogenic $E$. coli and $K$. pneumoniae which were circulating in this area with respect to the antibiogram. The prevalence of potential ESBL and MDR isolates were also explored.

\section{Materials and Methods}

\section{Study design and area}

This prospective study was conducted from urine samples collected from hospitalized patients complaining about UTIs. The samples were processed in Bacteriology Section, Department of Microbiology, Modern Diagnostic AND Research Centre, Gurugram, Haryana-India. 


\section{Processing of the samples}

All samples were processed within 1-2 hours of the collection, and in case of delay, the specimens were refrigerated at $4^{\circ} \mathrm{C}$. All urine samples were cultured by the semiquantitative method. In short, $0.01 \mathrm{ml}$ of urine was inoculated on Cysteine lactose electrolyte deficient agar (CLED Agar) (Hi-media Pvt. Ltd) by crisscross streaking using disposable calibrated flexi-loop (Hi-media Pvt. Ltd) and incubated for $18-24$ hours at $37^{\circ} \mathrm{C}$ under aerobic conditions.

A pure growth of the gram-negative isolate on a colony count $\geq 10^{5}$ colony forming units was considered as significant bacteriuria. Isolation and identification of the strains were done following their morphology in Gram's staining, culture characteristics and biochemical properties. Plates with no growth were further incubated for another 24 hours before interpreting it as a negative culture.

\section{Quality control}

The bacterial suspension was prepared and was adjusted to a $0.5 \mathrm{McFarland}$ standard solution (Hi-media Pvt. Ltd). American Type Culture Collection (ATCC) standard reference strains $P$. aeruginosa ATCC-27853, S.aureus ATCC-25923, E. coli ATCC-25922 were used as a quality control strains for antimicrobial susceptibility testing. All the ATCC strains used in the current prospective study were procured from Microbiologics, USA.

\section{Antibiotic sensitivity testing}

Antibiotic sensitivity testing was done by Kirby-Bauer disc diffusion method on Mueller-Hinton agar, and interpretation of the results was done as described by CLSI guidelines ${ }^{11}$.

Antibiotics discs (Hi-Media Lab Pvt. Ltd) used were Ampicillin (AMP)-10 $\mu \mathrm{g}$,
Gentamicin (GEN)-10 $\mu \mathrm{g}$, Tobramycin (TOB)-10 $\mu \mathrm{g}, \quad$ Amikacin (AK)-10 $\mu \mathrm{g}$, Amoxicillin-Clavulanic Acid (AMC)-20/10 $\mu \mathrm{g}$, Ampicillin/Sulbactum (A/S)-20/10 $\mu \mathrm{g}$, Piperacillin/Tazobactum (P/T)-100/10 $\mu \mathrm{g}$, Cefuroxime (CXM)-30 $\mu \mathrm{g}$, Cefepime (CPM)$30 \mu \mathrm{g}$, Ceftazidime (CAZ)-30 $\mu \mathrm{g}$, Aztreonam (AT)-15 $\mu \mathrm{g}, \quad$ Cefoxitin $\quad(\mathrm{CX})-30 \mu \mathrm{g}$, Levofloxacin (LE)- $5 \mu \mathrm{g}$, Ciprofloxacin (CIP)$5 \mu \mathrm{g}$, Imipenem (IMP)-10 $\mu \mathrm{g}$, Ertapenem (ETP)-10 $\mu \mathrm{g}$, Cotrimoxazole (COT)-25 $\mu \mathrm{g}$, Tetracycline (TET)-30 $\mu \mathrm{g}$, Nitrofurantoin (NIT) $-300 \mu \mathrm{g}$.

\section{Screening test for ESBL Production ${ }^{17}$}

The organism was swabbed onto the Mueller Hinton agar (MHA) plate. Antibiotic discs Ceftazidime with zone diameter of $\leq 22 \mathrm{~mm}$ and Cefotaxime with zone diameter of $\leq 27$ $\mathrm{mm}$ was considered indicative of suspicious for ESBL producer. These isolates were further subjected to the phenotypic confirmation testing ${ }^{11}$.

\section{Confirmatory test for ESBL production ${ }^{17}$}

ESBL productions among the potential ESBLproducing strains by screening method were confirmed by CLSI phenotypic confirmation method. Comparison of the zone of inhibition was made for the Ceftazidime $(30 \mu \mathrm{g})$ and Cefotaxime $(30 \mu \mathrm{g})$, discs alone with the Ceftazidime and Cefotaxime discs containing Clavulanic acid $(10 \mu \mathrm{g})$, ESBL production was confirmed by a $>5 \mathrm{~mm}$ increase in the zone diameter for either antibiotic tested in combination with Clavulanic acid against the zone diameter when tested alone confirmed the presence of ESBL production by that organism $^{11}$.

The increase in zone diameter was due to the inhibition of the $\beta$-lactamase by Clavulanate. K. pneumoniae (ATCC-700603) was used as a positive control, and E. coli (ATCC-25922) was used as a negative control. 


\section{Statistical analysis}

Chi-square test was used for statistical analysis of the data. A p-Value of less than 0.05 was considered as statistical significant.

\section{Results and Discussion}

\section{Demographical features of the studied subjects}

In the current study, 885 urine samples suspected of urinary tract infection were collected in sterile and leak-proof containers from hospitalized patients in and around Gurugram, Haryana. The median age was 54 years, and the age range was 0-92 years. There were $66.89 \% \quad(592 / 885) \quad$ culture-negative samples, with the median age of 52 years and age range of 0-92 years. In the present study, $33.11 \% \quad(293 / 885)$ samples were found positive for urine culture with the median age of 58 years and age range of $0-88$ years. Prevalence of urinary tract infection among the age wise distribution showed a significant difference $(\mathrm{p}=0.008)$ (Table 1). There were $52.43 \%(464 / 885)$ males included in this study and $79.74 \%(370 / 464)$ individuals presented with the negative urine culture. Rest of the $20.26 \%$ (94/464) males was presented with the positive urine culture. There were $47.57 \%$ (421/885) females included in the study and $52.73 \%$ (222/421) individuals presented with the negative urine culture. Rest of the $47.27 \%$ (199/421) females shown with the positive urine culture. Prevalence of urinary tract infection among the different gender showed a significant difference $(p=0.000)($ Table 1$)$.

\section{ESBL status of the uropathogens}

In the present study, Detection of ESBL was done by CLSI screening test followed by confirmatory tests ${ }^{11}$. It was performed for $E$. coli, K. pneumoniae and Proteus mirabilis ( $P$. mirabilis). Among these, only E. coli and $K$. pneumoniae were found to be positive. A total number of 237 gram-negative bacteria (Median age: 59; Range: 0-88) were isolated. Among them, 20.25\% (48/237) were ESBL producers (Median age: 58; Range: 0-80). and $43.88 \%$ (104/237) were non ESBL producers (Median age: 60 ; Range: 0-87) and $35.86 \%$ (85/237) were others isolates (Median age: 59; Range: 1-88). $44.73 \%$ (106/237) E. coli were isolated. The frequency of ESBL positive isolates was $35.85 \%$ (38/106), and ESBL negative isolates were $64.15 \%$ (68/106). $18.14 \% \quad(43 / 237) \quad K$. pneumoniae were isolated. The frequency of ESBL positive isolates were $23.26 \%(10 / 43)$, and ESBL negative isolates were $76.74 \%$ (33/43). $1.27 \%$ (3/237). Interestingly, all the $P$. mirabilis isolates were $\mathrm{ESBL}$ negative $(3,100 \%)$. In the current study, the frequency of ESBL negative uropathogens were significantly higher than ESBL positive strains $(p=0.046)$. Other GNB's were not tested for ESBL production (Table 2). There were $34.18 \%$ (81/237) males, in which $17.28 \%(14 / 81)$ isolates were ESBL positive, $37.04 \%$ (30/81) were ESBL negative and other GNB for which ESBL production was not tested were $45.68 \%$ (37/81). In females $65.82 \%$ (156/237), 21.79\% (34/156) isolates were ESBL positive, $47.44 \%$ (74/156) were ESBL negative, and other GNB for which ESBL production was not tested were $30.77 \%$ (48/156). Prevalence of ESBL production among the genders distribution was showed no significant difference $(\mathrm{p}=$ 0.076) (Table 2).

\section{Multidrug resistance patterns of ESBL $+\mathrm{Ve} / \mathrm{Ve}$ E. coli and K. pneumoniae}

All the ESBL producing isolates were 100\% resistant to all penicillin's, third-generation cephalosporins (e.g. ceftazidime, cefotaxime, and ceftriaxone) and Aztreonam. In ESBL positive $E$. coli $(\mathrm{n}=38)$, the most sensitive drug were Imipenem (97.0\%), Ertapenem (95.0\%), Nitrofurantoin (89.0\%) and Amikacin (82.0\%) and most resistant drugs Levofloxacin, Ciprofloxacin $(97.0 \%$ each), Tetracycline, 
Tobramycin, Ampicillin/Sulbactam $\quad(82.0 \%$ each), Cotrimoxazole (79.0\%) and Gentamicin (55.0\%) (Figure 1). In ESBL positive $K$. pneumoniae $(\mathrm{n}=10)$, the most sensitive drugs were Imipenem (100.0\%), Cefoxitin (90.0\%), Ertapenem (80.0\%), Tetracycline (50.0\%), and resistant to Cotrimoxazole, Ampicillin/ Sulbactam $(90.0 \%$ each), Ciprofloxacin, Tobramycin $(80.0 \%$ each) and Gentamicin (70.0\%) (Figure 2). In ESBL negative E. coli $(n=68)$ isolated from hospitalized patients, the most sensitive drug were Imipenem (81.0\%), Ertapenem (78.00\%), Amikacin, Nitrofurantoin $(74.0 \%$ each) and resistant to Ciprofloxacin (82.0\%), Levofloxacin (81.0\%), Ampicillin (76.0\%), Ampicillin/Sulbactam (65.0\%) (Figure 1). In ESBL negative $K$. pneumoniae $(\mathrm{n}=33)$, the strain was sensitive to Imipenem (45.0\%), Cefoxitin (39.0\%), Amikacin Piperacillin/Tazobactam, Tetracycline (36.0\% each), Amoxicillin/ clavulanic acid, Cefepime, Ceftazidime, Cefuroxime, Gentamicin, Levofloxacin, Nitrofurantoin, Tobramycin (30.0\% each) and Resistant to Ampicillin (100.0\%), Cotrimoxazole (73.0\%), Ampicillin/ Sulbactam, Cefepime, Aztreonam, Ceftazidime, Cefuroxime, Ciprofloxacin,
Gentamicin, Levofloxacin, Tobramycin (70.0\% each), Amoxicillin/clavulanic acid (64.0\%) (Figure 2). The sensitivity and resistance patterns of other antibiotics are showed in Figure 1 and 2.

In the current study, MDR was $82.55 \%$ $(123 / 149)$, and Non-MDR was $17.45 \%$ (26/149). The frequency of $E$. coli isolates were $71.14 \%$ (106/149), and $K$. pneumoniae were $28.86 \%$ (43/149). Among the 149 isolates, $32.21 \% \quad(48 / 149)$ were ESBL positive, and $67.79 \%$ (101/149) were ESBL negative. There were $84.91 \%(90 / 106)$ were MDR E. coli, and $15.09 \%$ (16/106) were nonMDR E. coli. There were $76.74 \%$ (33/43) MDR K. pneumoniae, and $23.26 \%$ (10/43) were non-MDR $K$. pneumoniae. In ESBL positive group $79.17 \%$ (38/48) were E. coli and $20.83 \%(10 / 48)$ were $K$. pneumoniae. ESBL positive $E$. coli and $K$. pneumoniae were found as MDR (100\%). In ESBL negative group, $67.33 \%$ (68/101) were E. coli and $32.67 \%$ (33/101) were $K$. pneumoniae. $76.47 \%$ (52/68) E. coli strains were MDR and $23.53 \% \quad(16 / 68)$ were non-MDR. 69.70\% (23/33) K. pneumoniae strains were MDR, and $30.30 \%$ (10/33) were non-MDR (Table 3).

Table.1 Demographical features of the studies subjects

\begin{tabular}{|c|c|c|c|c|}
\hline Features & TOTAL (n) & Neg* $(\%)$ & Pos** $(\%)$ & $p$-Value \\
\hline & 885 & $592(66.89$ & $293(33.11)$ & \multirow[t]{2}{*}{$\mathbf{N A}^{\#}$} \\
\hline MEDIAN AGE (RANGE) & $54(0-92)$ & $52(0-92)$ & $58(0-88)$ & \\
\hline \multicolumn{4}{|c|}{\begin{tabular}{l|l} 
AGE GROUP \\
\end{tabular}} & \multirow{5}{*}{0.008} \\
\hline $0-15$ & $77(8.70)$ & $50(64.94)$ & $27(35.06)$ & \\
\hline 16-30 & $135(15.25)$ & $106(78.52)$ & $29(21.48)$ & \\
\hline $31-45$ & $135(15.25)$ & $97(71.85)$ & $38(28.15)$ & \\
\hline 46-60 & $201(22.71)$ & $129(64.18)$ & $72(35.82)$ & \\
\hline$>60$ & $337(38.08)$ & $210(62.31)$ & $127(37.69)$ & \multirow{4}{*}{0.000} \\
\hline \multicolumn{4}{|l|}{ GENDER } & \\
\hline MALE & $464(52.43)$ & $370(79.74)$ & $94(20.26)$ & \\
\hline FEMALE & $421(47.57)$ & $222(52.73)$ & $199(47.27)$ & \\
\hline
\end{tabular}

*Neg- Negative, ${ }^{* * P o s}-$ Positive, ${ }^{\#} \mathrm{NA}$ - Not applicable 
Table.2 ESBL Status of Studied Uropathogens from hospitalized patients

\begin{tabular}{|c|c|c|c|c|c|}
\hline Features & $\begin{array}{c}\text { Total } \\
\text { GNB }\end{array}$ & ESBL+ve (\%) & ESBL-ve (\%) & $\begin{array}{l}\text { Other } \\
\text { GNB* }(\%)\end{array}$ & $p$-Value \\
\hline & 237 & $48(20.25)$ & $104(43.88)$ & $85(35.86)$ & \multirow{2}{*}{$\mathbf{N A}^{\#}$} \\
\hline MEDIAN AGE (RANGE) & $59(0-88)$ & $58(0-80)$ & $60(0-87)$ & $59(1-88)$ & \\
\hline \multicolumn{6}{|l|}{ ORGANISMS } \\
\hline E. coli & $106(44.73)$ & $38(35.85)$ & $68(64.15)$ & \multirow{5}{*}{$\mathbf{N A}^{\#}$} & \multirow{5}{*}{0.046} \\
\hline K. pneumoniae & $43(18.14)$ & $10(23.26)$ & $33(76.74)$ & & \\
\hline P.mirabilis & $3(1.27)$ & $0(0)$ & $3(100.0)$ & & \\
\hline K.oxytoca & NF** & NF** & NF** & & \\
\hline Others & $85(35.86)$ & $\mathbf{N A}^{\#}$ & $\mathbf{N A}^{\#}$ & & \\
\hline \multicolumn{6}{|l|}{ GENDER } \\
\hline MALE & $81(34.18)$ & $14(17.28)$ & $30(37.04)$ & $37(45.68)$ & \multirow{2}{*}{0.076} \\
\hline FEMALE & $156(65.82)$ & $34(21.79)$ & $74(47.44)$ & $48(30.77)$ & \\
\hline
\end{tabular}

*GNB- Gram Negative Bacteria, "NA - Not applicable, NF**- Not found

Table.3 MDR Status of ESBL +/-ve E. coli and K. pneumoniae isolates from hospitalized patients

\begin{tabular}{|l|l|l|l|}
\hline Features & Total $(\%)$ & MDR* $(\%)$ & NON-MDR* $(\%)$ \\
\hline ORGANISMS & 149 & $123(82.55)$ & $26(17.45)$ \\
\hline E. coli & $106(71.14)$ & $90(84.91)$ & $16(15.09)$ \\
\hline K. pneumoniae & $43(28.86)$ & $33(76.74)$ & $10(23.26)$ \\
\hline ESBL POSITIVE & $\mathbf{4 8 ( 3 2 . 2 1 )}$ & $\mathbf{4 8 ( 1 0 0 . 0 )}$ & $\mathbf{0}(\mathbf{0})$ \\
\hline E. coli & $38(79.17)$ & $38(100.0)$ & $0(0)$ \\
\hline K. pneumoniae & $10(20.83)$ & $10(100.0)$ & $0(0)$ \\
\hline ESBL NEGATIVE & $\mathbf{1 0 1}(\mathbf{6 7 . 7 9})$ & $\mathbf{7 5 ( 7 4 . 2 6 )}$ & $\mathbf{2 6}(\mathbf{2 5 . 7 4 )}$ \\
\hline E. coli & $68(67.33)$ & $52(76.47)$ & $16(23.53)$ \\
\hline K. pneumoniae & $33(32.67)$ & $23(69.70)$ & $10(30.30)$ \\
\hline
\end{tabular}

*MDR- Multi-Drug Resistant.

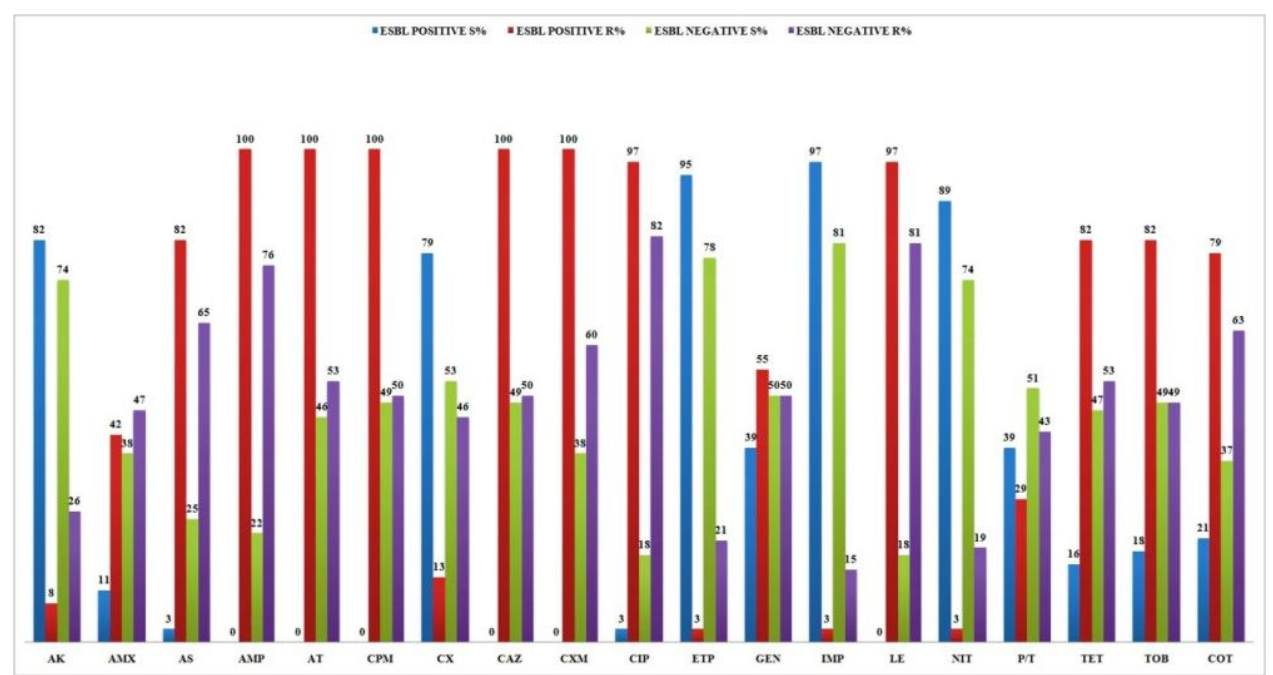

Fig.1 Antibiogram showing Sensitivity and Resistance Patterns of E. coli in hospitalized patients $\mathrm{S} \%=$ Sensitivity; $\mathrm{R} \%=$ Resistant 


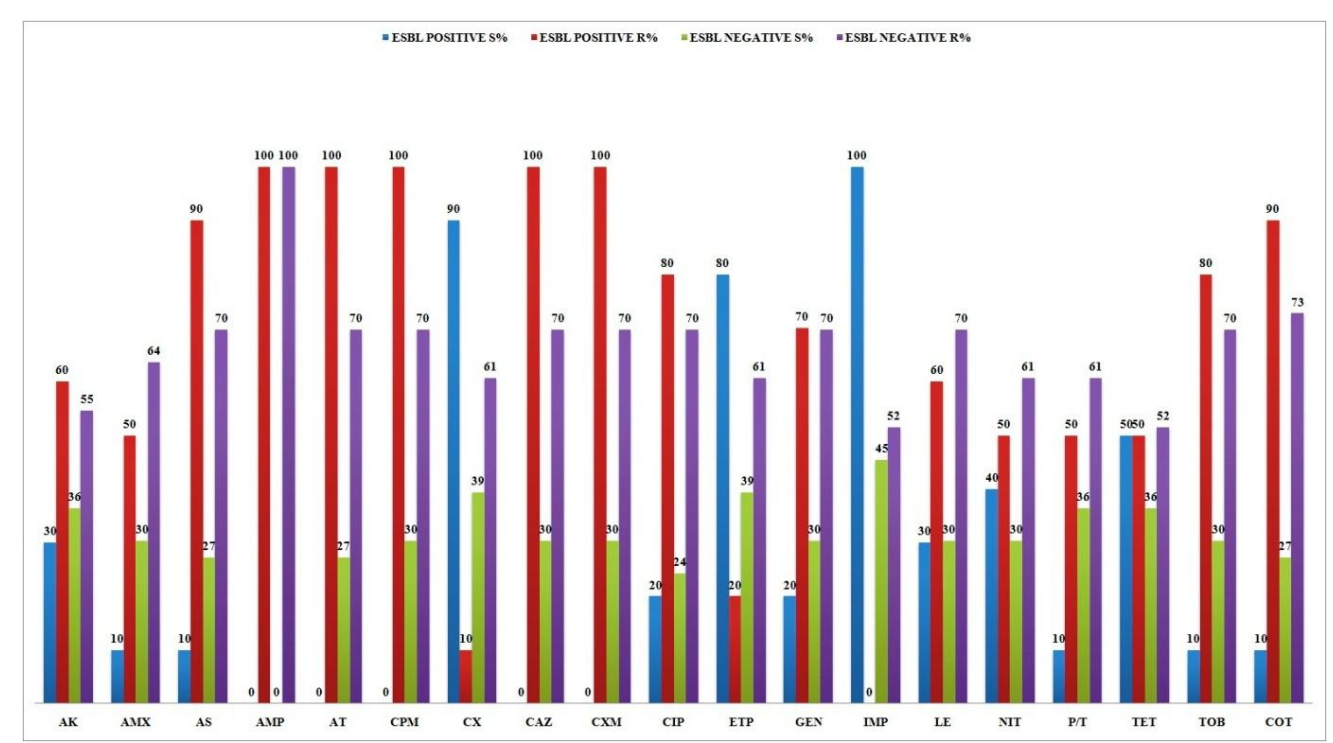

Fig.2 Antibiogram showing Sensitivity and Resistance Patterns of $K$. pneumoniae in hospitalized patients $\mathrm{S} \%=$ Sensitivity; R\% = Resistant

The present study evaluated the antibiogram of the ESBL producing and non-producing strains isolated from the hospitalized patients from in and around Gurugram, Haryana. To the best of our knowledge, this is the first research from our area with an exclusive focus on investigating the current prevalence and antibiogram among ESBL producing E. coli and $K$. pneumoniae the predominant uropathogens, globally.

Urinary tract infection is known as the one of the most common infection in patients with hospitalization ${ }^{1}$. The spread of drug resistance among gram-negative bacteria leads to the appearance of strains resistant to the antibiotics. These strains cause the development of UTIs resistant to the antibiotic therapy $^{12}$.

The positive culture rate with significant colony counts found among hospitalized patients was $33.11 \%$. In the other study done at Jaipur showed $41.8 \%{ }^{13}$ which was higher than our study and similar higher results were seen in various other studies ${ }^{14-16}$. The antibiotic sensitivity pattern has changed over time and in different areas, but the spectrum of agents causing Urinary tract infection has remained comparatively invariable, with $E$. coli being the most common isolate ${ }^{17}$. In the current study, females were found to be more infected with the urinary tract infection as compared to males (Females:47.27 \%; Males:20.26\%, pvalue: 0.000$)$. The findings were contradictory to the study done by Bajpai et al., and Singhal et al., which found male were more prevalent in UTI as compared to females ${ }^{13,18}$. These findings were in line with the other studies done by different authors in different regions ${ }^{10,19,20}$.

In the present study, E. coli was the most common isolate $(44.73 \%)$. This was similar to the studies conducted by other researchers ${ }^{16,21,22}$. However, studies from some other parts of the country had shown higher frequency rates $(65-90 \%)^{23-25}$. This variation might be due to the different environmental conditions in different areas. Prevalence of $K$. pneumoniae in hospitalized patients was $18.14 \%$ which was higher than the study conducted by Singhal et al. $(11.0 \%)^{13}$.

Prevalence of total ESBL production among all analysed uropathogens in the current study was $20.25 \%$ whereas lesser than the study conducted by Khurana et al. (26.6\%) and 
Tankhiwale et al. $(48.3 \%)^{26,27}$. In the present study reported $35.85 \%$ E. coli ESBL positive isolates resembles the study published by Kumar et al. $(39.0 \%)^{28}$ and unlike those reported by Singhal et al. (62.0\%), Maya et al. $(75.5 \%)$ and Ramesh et al. $(60.7 \%)^{13,29,30}$. $23.26 \%$ of the $K$. pneumoniae strains were found to be ESBL producer which is closer to the study done in Algeria $(20.0 \%)^{31}$. In Pakistan, the prevalence was $36 \%^{32}$. In India, $68 \%$ were ESBL producing ${ }^{33}$. Studies have also shown an increase in ESBL- producing strains of $K$. pneumoniae, $29 \%$ in Spain ${ }^{34}$, $28.4 \%$ in Taiwan ${ }^{35}$ and $44 \%$ in the $\mathrm{USA}^{36}$. In light of the above findings, it is clear that the prevalence of a wide variety of ESBL enzymes is rising. It seems that increased and prolonged duration of hospitalization and treatment procedures, as well as frequent use of urinary catheters in hospitals, are responsible for the emergence of resistant strains and transfer of resistant genes to other bacteria.

Our study confirms the Global trend towards the increased resistance to the $\beta$-lactam group of antibiotics. ESBL producing bacteria may not be detectable by routine disc diffusion sensitivity testing, leading to the inappropriate use of antibiotics and failure in the treatment. It was emphasized that Institutions should employ appropriate tests for their detection and avoid indiscriminate use of third-generation cephalosporins. The treatment should only be given after the culture and sensitivity test report to prevent the MDR.

Antibiotic resistance showed by different isolates is one of the barricades that might hinder a successful treatment. Widespread use of antibiotics exerts the selective pressure that acts as a driving force in the development of the resistance to various antibiotics ${ }^{18}$. The detailed insight of the antibiogram is illustrated in Figure 1 and 2. Constant survey of the antibiotic-resistant pattern plays a very crucial role in the empiric treatment of UTIs.
In the present study, all ESBL positive E. coli and $K$. pneumoniae were found to be MDR due to which the treatment options remained very limited in the hospital settings. In comparison with other studies done, A very high MDR rates of $91.66 \%$ and $87.5 \%$ among ESBL positive E. coli and $K$. pneumoniae were obtained in this study ${ }^{37}$. A comparable MDR rate of $83 \%$ among E. coli isolates was reported in a study done in Peshawar, Pakistan $^{38}$.

In the study, $97 \%$ sensitive to ESBL positive isolates and $100 \%$ in ESBL positive $K$. pneumoniae isolates. Hence carbapenems can be the alternative treatment to the thirdgeneration cephalosporins, fluoroquinolones and MDR strains ( $\geq$ 3antimicrobial classes) in the hospitalized patients due to ESBL positive uropathogens. In comparison to the study conducted by Aggarwal et al. 100\% Imipenem sensitivity was observed ${ }^{4}$. Similar kind of findings were observed in the study conducted by different authors ${ }^{39-42}$.

In earlier studies, prolonged hospitalization, Foley's catheterization, prior surgery, and ICU stay were found to be risk factors ${ }^{43-45}$. Good infection control practices and antibiotic management interventions are instrumental in preventing the emergence of outbreaks due to ESBL producing isolates, especially in highrisk areas such as the medical ICU, the neonatal ICU, and oncology units. Educational programs for medical, nursing and another supporting hospital to increase awareness of ESBLs should also be developed.

This study revealed the resistant rates of $70.0 \%$ and $60 \%$ to the aminoglycosides, Gentamicin and Amikacin by ESBL positive $K$. pneumoniae isolates. In comparison, E. coli shown a much low resistance rate: Gentamicin $55.0 \%$; Amikacin: $8.0 \%$. In contrast, $K$. pneumoniae showed resistance to Gentamicin $(69 \%)$ and Amikacin (38\%) while 59\% and 
$33 \%$ resistance rates were confirmed by $E$. coli isolates in a study done in Indore, India ${ }^{46}$. A resistance rate of $46.7 \%$ to gentamicin was demonstrated by $K$. pneumoniae isolates in a report from Karachi, Pakistan ${ }^{47}$, which is similar to results in this study. This release supports that aminoglycosides have excellent activity against clinically significant gramnegative bacilli ${ }^{48}$. The resistance rates of ESBL positive $E$. coli and $K$. pneumoniae to nitrofurantoin were $3.0 \%$ and $50.0 \%$, respectively. Resistance rate (12\%) by $E$. coli isolates was shown in a report from Indore, India $^{46}$.

In conclusion, E. coli and K. pneumoniae were the most predominant uropathogens and ESBL positive due to which MDR has been increased. MDR to commonly used antimicrobials in uropathogens has caused considerable alarm which suggests the importance of judicious use of antimicrobials. Carbapenems were the most promising drugs against Gram-negative bacilli. Nitrofurantoin can be considered as the alternative option in the empirical treatment of UTI. It is suggested to not to start any antibiotics without the culture and sensitivity report.

In the present study, the global trend toward increased resistance to $\beta$-lactam antibiotics was confirmed. It was emphasized that institutions with a high prevalence of third-generation cephalosporin resistant organisms should employ appropriate tests for their detection and avoid indiscriminate use of third-generation cephalosporins. Also, the incidence and antibiogram of ESBL producers differ geographically. Hence, such institutional studies will help in the formulation of antibiotic policy for a particular geographical area.

The strict antibiotic policy should be adopted in hospitals to estimate the impact of higher resistance in bacteria and to take steps for reducing the resistance. There are several possible methods for overcoming resistance including reduced use of antibiotics, use of synergistic combinations, the addition of an anti-resistance factor, attacking the underlying disease, improving the hygienic measures and regular surveillance studies.

This report documents the emergence and occurrence of ESBL producing E. coli and $K$. pneumoniae in urinary isolates in our area. A high prevalence of ESBL producing E. coli and $K$. pneumoniae was observed and confirmed in the urinary isolates. A strict hospital infection control policies and prudent antimicrobials use regimens to be adopted by the physicians. It is essential and mandatory to have regular and routine monitoring of ESBL producing clinical isolates in clinical laboratories.

\section{Acknowledgements}

The study was done at Bacteriology section, Department of Microbiology, Modern Diagnostic and Research Centre, Gurugram. We would like to express our special appreciation and thanks to all the patients whose samples were used in this study, my newborn angel Ivanka Rajput, wife and fathermother who gave me inspiration to write the research papers, technicians of my department, my beloved friends Dr. Ajay Soni, Dr. Siva Adarsh, Dr. Sangeet Bhaumik and our Director, Dr. D.S Yadav for his continuous guidance, constitutive criticism, and constant encouragement.

\section{References}

1. Das, R. N. et al. Frequency and susceptibility profile of pathogens causing urinary tract infections at a tertiary care hospital in western Nepal. Singapore Med. J. 47, 281-5 (2006).

2. Barguigua, A. et al. Characterization of extended-spectrum -lactamase-producing 
E. coli and $K$. pneumoniae isolates from the community in Morocco. J. Med. Microbiol. 60, 1344-1352 (2011).

3. Latifpour, M., Gholipour, A. and Damavandi, M. S. Prevalence of ExtendedSpectrum Beta-Lactamase-Producing $K$. pneumoniae Isolates in Nosocomial and Community-Acquired Urinary Tract Infections. Jundishapur J. Microbiol. 9, (2016).

4. Aggarwal, R., Chaudhary, U. and Sikka, R. Detection of Extended Spectrum $\beta$ lactamase Production Among Uropathogens. J. Lab. Physicians 1, 7-10 (2009).

5. Bradford, P. A. Extended-Spectrum Lactamases in the 21st Century: Characterization, Epidemiology, and Detection of This Important Resistance Threat. Clin. Microbiol. Rev. 14, 933-951 (2001).

6. Livermore, D. M. beta-lactamases in laboratory and clinical resistance. Clinical Microbiology Reviews 8, 557-584 (1995).

7. Eftekhar, F., Rastegar, M., Golalipoor, M. and Mansoursamaei, N. Detection of Extended Spectrum B-Lactamases in Urinary Isolates of $K$. pneumoniae in Relation to Bla, Bla and Bla Gene Carriage. Iran. J. Public Health 41, 127-32 (2012).

8. Shaikh, S., Fatima, J., Shakil, S. and Rizvi, S. M. D. Antibiotic resistance and extended spectrum beta-lactamases: Types, epidemiology and treatment. Saudi J. Biol. Sci. 22, 90-101 (2015).

9. Chaudhary, U. and Aggarwal, R. Extended spectrum -lactamases (ESBL) - an emerging threat to clinical therapeutics. Indian J. Med. Microbiol. 22, 75-80 (2004).

10.Sood, S. and Gupta, R. Antibiotic resistance pattern of community acquired uropathogens at a tertiary care hospital in jaipur, rajasthan. Indian J. Community Med. 37, 39-44 (2012).

11.CLSI. Performance Standards for
Antimicrobial Susceptibility Testing; Nineteenth Informational Supplement. CLSI document M100-S19. Wayne, PA. USA. 29, (2009).

12.Blondeau, J. M. and Vaughan, D. A review of antimicrobial resistance in Canada. Can. J. Microbiol. 46, 867-77 (2000).

13.Singhal, A., Sharma, R., Jain, M. and Vyas, L. Hospital and Community Isolates of Uropathogens and their Antibiotic Sensitivity Pattern from a Tertiary Care Hospital in North West India. Ann. Med. Health Sci. Res. 4, 51-6 (2014).

14.Arjunan, M., Al-Salamah, A. A. and Amuthan, M. Prevalence and Antibiotics Susceptibility of Uropathogens in Patients from a Rural Environment. Am. J. Infect. Dis. 6, 29-33 (2010).

15.Aypak, C., Altunsoy, A. and Düzgün, N. Empiric antibiotic therapy in acute uncomplicated urinary tract infections and fluoroquinolone resistance: a prospective observational study. Ann. Clin. Microbiol. Antimicrob. 8, 27 (2009).

16.Gupta, V., Yadav, A. and Joshi, R. M. Antibiotic resistance pattern in uropathogens. Indian J. Med. Microbiol. 20, 96-8 (2002).

17.Gupta, K., Hooton, T. M. and Stamm, W. E. Increasing antimicrobial resistance and the management of uncomplicated community-acquired urinary tract infections. Ann. Intern. Med. 135, 41-50 (2001).

18.Bajpai, T., Pandey, M., Varma, M. and Bhatambare, G. S. Prevalence of extended spectrum beta-lactamase producing uropathogens and their antibiotic resistance profile in patients visiting a tertiary care hospital in central India: Implications on empiric therapy. Indian J. Pathol. Microbiol. 57, 407-12 (2014).

19. Sasirekha, B. Prevalence of ESBL, AmpC $\beta$-lactamases and MRSA among uropathogens and its antibiogram. EXCLI J. 12, 81-8 (2013). 
20.Manjunath GN, Prakash R, Vamseedhar Annam, K. S. Changing trends in the spectrum of antimicrobial drug resistance pattern of uropathogens isolated from hospitals and community patients with urinary tract infections in Tumkur and. Int. J. Biol. Med. Res. 2, 504-507 (2011).

21.Kamat, U. S., Fereirra, A., Amonkar, D., Motghare, D. D. and Kulkarni, M. S. Epidemiology of hospital acquired urinary tract infections in a medical college hospital in Goa. Indian J. Urol. 25, 76-80 (2009).

22.Taneja, N., Chatterjee, S. S., Singh, M., Singh, S. and Sharma, M. Pediatric urinary tract infections in a tertiary care center from north India. Indian J. Med. Res. 131, 101-5 (2010).

23. Chatterjee, B., Kulathinal, S., Bhargava, A., Jain, Y. and Kataria, R. Anti-microbial resistance stratified by risk factor among $E$. coli strains isolated from the urinary tract at a rural clinic in Central India. Indian $J$. Med. Microbiol. 27, 329-34 (2009).

24.Kadri, S. M., Gash, B. and Rukhsana, A. Antibiotic sensitivity and resistance profile of the micro-organisms responsible for urinary tract infection observed in Kashmir, India. J. Indian Med. Assoc. 100, 656, 65860 (2002).

25.Akram, M., Shahid, M. and Khan, A. U. Etiology and antibiotic resistance patterns of community-acquired urinary tract infections in J N M C Hospital Aligarh, India. Ann. Clin. Microbiol. Antimicrob. 6, 4 (2007).

26.Khurana, S., Taneja, N. and Sharma, M. Extended spectrum beta-lactamase mediated resistance in urinary tract isolates of family Enterobacteriaceae. Indian $J$. Med. Res. 116, 145-9 (2002).

27. Tankhiwale, S. S., Jalgaonkar, S. V, Ahamad, S. and Hassani, U. Evaluation of extended spectrum beta lactamase in urinary isolates. Indian J. Med. Res. 120, 553-6 (2004).

28.Kumar, M. S., Lakshmi, V. and
Rajagopalan, R. Occurrence of extended spectrum beta-lactamases among Enterobacteriaceae spp. isolated at a tertiary care institute. Indian J. Med. Microbiol. 24, 208-11 (2006).

29. Maya AS, Prabhakar K, Hanna LE, S. Y. Phenotypic and Genotypic Characterization of Extended Spectrum b-Lactamase Producing E. coli Clinical Isolates from Semiurban Area. J. Pharm. Res. 44, 6-10 (2011).

30.Ramesh N, Sumathi CS, Balasubramanian V, Palaniappan KR, K. V. Urinary Tract Infection and Antimicrobial Susceptibility Pattern of Extended Spectrum of Beta Lactamase Producing Clinical Isolates. $A d v$. Biol ogical Res. 2, 78-82 (2008).

31. Messai, Y. et al. Prevalence and characterization of extended-spectrum $\beta$ lactamases in $K$. pneumoniae in Algiers hospitals (Algeria). Pathol. Biol. 56, 319325 (2008).

32.Jabeen, K., Zafar, A. and Hasan, R. Frequency and sensitivity pattern of Extended Spectrum beta Lactamase producing isolates in a tertiary care hospital laboratory of Pakistan. J. Pak. Med. Assoc. 55, 436-9 (2005).

33. Shanthi $\mathrm{J}$ and $\mathrm{S}, \mathrm{K}$. Incidence, distribution and antibiogram of uropathogens isolated from patients with urinary tract infections. Pelagia Res. Libr. Adv. Appl. Sci. Res. 3, 3410-3414 (2012).

34.Romero, E. D. V. et al. Prevalence of clinical isolates of E. coli and Klebsiella spp. producing multiple extended-spectrum beta-lactamases. Diagn. Microbiol. Infect. Dis. 59, 433-7 (2007).

35.Kuo, K. C., Shen, Y. H. and Hwang, K. P. Clinical implications and risk factors of extended-spectrum beta-lactamaseproducing $K$. pneumoniae infection in children: a case-control retrospective study in a medical center in southern Taiwan. $J$. Microbiol. Immunol. Infect. 40, 248-54 (2007). 
36.Saurina, G., Quale, J. M., Manikal, V. M., Oydna, E. and Landman, D. Antimicrobial resistance in Enterobacteriaceae in Brooklyn, NY: epidemiology and relation to antibiotic usage patterns.1. Saurina, G., Quale, J. M., Manikal, V. M., Oydna, E. and Landman, D. Antimicrobial resistance in Enterobacteriaceae in Brooklyn, NY: ep. J. Antimicrob. Chemother. 45, 895-8 (2000).

37. Chander, A. and Shrestha, C. D. Prevalence of extended spectrum beta lactamase producing $E$. coli and $K$. pneumoniae urinary isolates in a tertiary care hospital in Kathmandu, Nepal. BMC Res. Notes 6, 487 (2013).

38.Ullah, F., Malik, S. and Ahmed, J. Antibiotic susceptibility pattern and ESBL prevalence in nosocomial $E$. coli from urinary tract infections in Pakistan. African J. Biotechnol. 8, 3921-26 (2002).

39. Baby Padmini, S., Appala Raju, B. and Mani, K. R. Detection of Enterobacteriaceae producing CTX-M extended spectrum beta-lactamases from a tertiary care hospital in south India. Indian J. Med. Microbiol. 26, 163-6

40.Shukla, I., Tiwari, R. and Agrawal, M. Prevalence of extended spectrum lactamase producing $K$. pneumoniae in a tertiary care hospital. Indian J. Med. Microbiol. 22, 87-91 (2004).

41.Peña, C. et al. Epidemiology and successful control of a large outbreak due to $K$. pneumoniae producing extended-spectrum beta-lactamases. Antimicrob. Agents Chemother. 42, 53-8 (1998).

\section{How to cite this article:}

Rishabh Rajput and Surendra Sarsaiya. 2018. Multi-drug Resistance and Extended-Spectrum $\beta$ Lactamase Production in Uropathogens from Hospitalized Patients in Gurugram, India. Int.J.Curr.Microbiol.App.Sci. 7(02): 1270-1281. doi: https://doi.org/10.20546/ijcmas.2018.702.155
42.Hansotia, J. B., Agarwal, V., Pathak, A. A. and Saoji, A. M. Extended spectrum betalactamase mediated resistance to third generation cephalosporins in $K$. pneumoniae in Nagpur, central India. Indian J. Med. Res. 105, 158-61 (1997).

43.Paterson, D. L. and Bonomo, R. A. Extended-spectrum beta-lactamases: a clinical update. Clin. Microbiol. Rev. 18, 657-86 (2005).

44.Rupp, M. E. and Fey, P. D. Extended spectrum beta-lactamase (ESBL)-producing Enterobacteriaceae: considerations for diagnosis, prevention and drug treatment. Drugs 63, 353-65 (2003).

45. Nathisuwan, S., Burgess, D. S. and Lewis, J. S. Extended-spectrum beta-lactamases: epidemiology, detection, and treatment. Pharmacotherapy 21, 920-8 (2001).

46.Pathak, A. et al. High prevalence of extended-spectrum andamp; beta;lactamase-producing pathogens: results of a surveillance study in two hospitals in Ujjain, India. Infect. Drug Resist. 65 (2012). doi:10.2147/IDR.S30043

47. Abdullah, F. E. et al. Current efficacy of antibiotics against Klebsiella isolates from urine samples - a multi-centric experience in Karachi. Pak. J. Pharm. Sci. 26, 11-5 (2013).

48.American Academy of Family Physicians., L. S. G. and Spencer, J. P. Aminoglycosides: A Practical Review. American Family Physician 58, (American Academy of Family Physicians, 1970). 